\title{
A Simple Proof of the Multinomial Theorem using Multivariate Calculus
}

\author{
Sumit Kumar Jha \\ IIIT-Hyderabad, India \\ Email: kumarjha.sumit@research.iiit.ac.in
}

May 16, 2019

Let $m, n$ be non-negative integers and $x_{1}, x_{2}, \cdots, x_{m}$ be real numbers, then the multinomial theorem tells us that

$$
f=\left(x_{1}+x_{2}+\cdots+x_{m}\right)^{n}=\sum_{k_{1}+k_{2}+\cdots+k_{m}=n} \frac{n !}{k_{1} ! k_{2} ! \cdots k_{m} !} x_{1}^{k_{1}} x_{2}^{k_{2}} \cdots x_{m}^{k_{m}} .
$$

Now we proceed with the proof. Applying the distributive law and collecting terms, we can see that

$$
\left(x_{1}+x_{2}+\cdots+x_{m}\right)^{n}=\sum_{k_{1}+\cdots+k_{m}=n} C\left(n, k_{1}, \cdots, k_{m}\right) x_{1}^{k_{1}} x_{2}^{k_{2}} \cdots x_{m}^{k_{m}},
$$

where $C\left(n, k_{1}, \cdots, k_{m}\right)$ denotes the coefficient of $x_{1}^{k_{1}} x_{2}^{k_{2}} \cdots x_{m}^{k_{m}}\left(\sum_{i=1}^{m} k_{i}=n\right)$ in the expansion. Now, observe that

$$
C\left(n, k_{1}, \cdots, k_{m}\right)=\frac{1}{k_{1} ! k_{2} ! \cdots k_{m} !}\left[\frac{\partial^{n} f}{\partial x_{1}^{k_{1}} \partial x_{2}^{k_{2}} \cdots \partial x_{m}^{k_{m}}}\right]_{x_{1}, x_{2}, \cdots, x_{m}=0},
$$

that is, in words, we first differentiate $f$ exactly $k_{1}$ times with respect to $x_{1}$, divide by $k_{1}$ !, then differentiate further exactly $k_{2}$ times with respect to $x_{2}$ divide by $k_{2}$ !, and so on until differentiating with respect to $x_{m}$ exactly $k_{m}$ times and divide by $k_{m}$ !; and to finally make sure we get desired coefficient plug all $x_{1}, \cdots, x_{m}$ to be 0 .

But observe that

$$
\begin{gathered}
\frac{\partial^{k_{1}} f}{\partial x_{1}^{k_{1}}}=(n)(n-1) \cdots\left(n-k_{1}+1\right) \cdot\left(x_{1}+\cdots+x_{m}\right)^{n-k_{1}} \\
\frac{\partial^{k_{1}+k_{2}} f}{\partial x_{2}^{k_{2}} \partial x_{1}^{k_{1}}}=(n)(n-1) \cdots\left(n-\left(k_{1}+k_{2}\right)+1\right) \cdot\left(x_{1}+\cdots+x_{m}\right)^{n-k_{1}-k_{2}} \\
\vdots \\
\frac{\partial^{k_{1}+\cdots+k_{m}} f}{\partial x_{1}^{k_{1}} \partial x_{2}^{k_{2}} \cdots \partial x_{m}^{k_{m}}}=n ! \cdot\left(x_{1}+\cdots+x_{m}\right)^{0}=n ! .
\end{gathered}
$$

This completes the proof. 